\title{
Closing the detection loophole in multipartite Bell tests using Greenberger-Horne-Zeilinger states
}

\author{
Károly F. Pál, ${ }^{1}$ Tamás Vértesi, ${ }^{1}$ and Nicolas Brunner ${ }^{2,3}$ \\ ${ }^{1}$ Institute of Nuclear Research of the Hungarian Academy of Sciences, H-4001 Debrecen, P.O. Box 51, Hungary \\ ${ }^{2}$ Département de Physique Théorique, Université de Genève, 1211 Genève, Switzerland \\ ${ }^{3}$ H.H. Wills Physics Laboratory, University of Bristol, Tyndall Avenue, Bristol, BS8 1TL, United Kingdom
}

(Received 7 August 2012; published 19 December 2012)

\begin{abstract}
We investigate the problem of closing the detection loophole in multipartite Bell tests, and show that the required detection efficiencies can be significantly lowered compared to the bipartite case. In particular, we present Bell tests based on $n$-qubit Greenberger-Horne-Zeilinger states, which can tolerate efficiencies as low as $38 \%$ for a reasonable number of parties and measurements. Even in the presence of a significant amount of noise, efficiencies below 50\% can be tolerated, which is encouraging given recent experimental progress. Finally, we give strong evidence that, for a sufficiently large number of parties and measurements, arbitrarily small efficiencies can be tolerated, even in the presence of an arbitrary large amount of noise.
\end{abstract}

DOI: 10.1103/PhysRevA.86.062111

PACS number(s): 03.65.Ud

Quantum nonlocality is arguably one of the most counterintuitive aspects of quantum mechanics. According to quantum theory, separated parties sharing an entangled state and performing suitably chosen measurements are able to generate correlations which are unexplainable by any classical mechanism. These nonlocal correlations can be tested experimentally using Bell inequalities [1]. Numerous experiments have demonstrated Bell inequality violations giving strong evidence that nature is inherently nonlocal [2]. However, technical imperfections in these experiments open various loopholes, which make it still possible to explain the data with a local model. Given the fundamental importance of nonlocality, it is highly desirable to perform a loophole-free Bell test, which, despite recent theoretical proposals (see, e.g., [3]) and experimental progress [4,5], is still missing.

A loophole-free Bell test requires (i) a spacelike separation between the parties and (ii) a detection efficiency above a certain threshold (usually high). The first condition ensures that no communication between the parties is possible, hence closing the locality loophole. This was achieved in photonic experiments [2]. The second condition ensures that no classical model exploiting undetected events can reproduce the observed data, hence closing the detection loophole [6]. This was achieved in atomic experiments [7]. However, no experiments could yet close both loopholes simultaneously. On the one hand, atomic experiments are unsatisfactory from the locality point of view. On the other hand, typical photodetection efficiencies are still too low to close the detection loophole.

Addressing the detection loophole is also crucial for information-theoretic applications based on quantum nonlocality [8-10]. Failure in closing the detection loophole renders these protocols insecure as the observed Bell violation may have been produced by classical means, as nicely illustrated by recent experiments faking Bell violations [11].

In general, the required detection efficiency $\eta$ depends on the Bell inequality and the quantum state which are considered. For the Clauser-Horne-Shimony-Holt (CHSH) inequality, an efficiency $\eta>82.8 \%$ is required for a maximally entangled qubit pair, while $\eta>66.7 \%$ for a partially entangled state [12]. More recently, improvements were reported using four- dimensional quantum systems, tolerating efficiencies $\sim 61 \%$ [13]. However, from a practical point of view, these results should be considered carefully, in particular when taking into account additional imperfections such as background noise. Importantly, even a small amount of noise increases significantly the threshold efficiencies; in the CHSH case for instance, adding $1 \%$ of noise to the state increases the threshold from $66.7 \%$ to $80 \%$ [12].

Another approach, which has received so far only little attention, is to consider multipartite Bell tests, that is, with $n>2$ observers. Based on a combinatorial study, Buhrman et al. [14] showed that an arbitrarily small efficiency $\eta$ can be tolerated as $n$ becomes large. More recently, threshold efficiencies for the Mermin inequalities were shown to approach $\eta=50 \%$ for large $n$ [15], but remain above $60 \%$ for any practical scenario. The same limit can be approached for the many-site generalization of the Clauser-Horne inequality [16]. Also, a multipartite Bell test based on single-photon entanglement was shown to approach $\eta=66.7 \%$ for large $n$ [17]. However, up until now, no practical Bell test featuring efficiencies lower than $60 \%$ for all observers was known [18].

Here we show that detection efficiencies as low as $38 \%$ can be tolerated in multipartite Bell tests featuring a reasonable number of parties and measurements, and lower than $50 \%$ even in the presence of noise. Specifically, we present a family of Bell tests, based on Bell inequalities, in which $n$ observers perform $m$ binary measurements on an $n$-qubit GreenbergerHorne-Zeilinger (GHZ) state [20]. Notably, efficiencies $\eta<$ $50 \%$ can be tolerated already for a 6-qubit GHZ state and $m=7$ or alternatively for a 5-qubit GHZ state and $m=11$. Furthermore, the measurements to be performed are equally distributed on an equator of the Bloch sphere, which is convenient from a practical point of view. Moreover our Bell tests appear to be robust to noise. For instance, for an 8-qubit GHZ state with $10 \%$ of noise, efficiencies $\eta \sim 50 \%$ can be tolerated for $m=7$. From an experimental perspective, these results look encouraging, given recent experimental progress [21], in particular the observation of 8-qubit GHZ states [22]. Finally, we investigate the efficiency for our Bell tests in the asymptotic limit. We give strong evidence that $\eta \rightarrow 2 / n$ when $m \rightarrow \infty$ for a pure GHZ state. Moreover, we give evidence 
that arbitrarily low efficiencies can be tolerated, even if an arbitrary amount of noise is added to the GHZ state.

\section{SETUP}

We consider a Bell scenario with $n$ distant observers. Each observer may choose between a set of $m$ measurements $\left\{A_{i}\right\}$, $\left\{B_{j}\right\},\left\{C_{k}\right\}$, and so on, with $i, j, k, \ldots=0, \ldots, m-1$. All measurements have binary outcomes, +1 and -1 . We use the shorthand notation $P\left(A_{i} B_{j} C_{k} \cdots\right) \equiv P\left(111 \cdots \mid A_{i} B_{j} C_{k} \cdots\right)$ and similarly for any subset of parties. We start by defining a family of Bell inequalities:

$$
\begin{aligned}
& \sum_{i, j, k, \ldots=0}^{m-1} P\left(A_{i} B_{j} C_{k} \cdots\right)\left(y-x \delta_{(i+j+k+\cdots) \bmod m}^{0}\right) \\
& -\sum_{j, k, \ldots=0}^{m-1} P\left(B_{j} C_{k} \cdots\right)-\sum_{i, k, \ldots=0}^{m-1} P\left(A_{i} C_{k} \cdots\right) \\
& \quad-\sum_{i, j, \ldots=0}^{m-1} P\left(A_{i} B_{j} \cdots\right)-\cdots \leqslant 0
\end{aligned}
$$

where $\delta_{x \bmod m}^{0}=1$ if $x$ is divisible by $m$, and is 0 otherwise. Note that the real parameters $x$ and $y$ are chosen such that the local bound of the Bell inequality is 0 . We shall see later how this condition can be enforced.

The observers share a noisy $n$-qubit GHZ state

$$
\hat{\rho}=v|\mathrm{GHZ}\rangle\langle\mathrm{GHZ}|+(1-v) \frac{\mathbb{1}}{2^{n}},
$$

with $|\mathrm{GHZ}\rangle \equiv\left(|0\rangle^{\otimes n}+|1\rangle^{\otimes n}\right) / \sqrt{2}$ and $v$ is the visibility. This state is fully separable iff $v \leqslant 1 /\left(1+2^{n-1}\right)$ [23] and violates a two-setting full-correlation inequality for $v>1 / 2^{(n-1) / 2}$ [24].

Here we will focus on (projective) equatorial qubit measurements of the form

$$
\begin{aligned}
& \hat{A}_{i}=\cos \varphi_{i}^{A} \hat{\sigma}_{x}+\sin \varphi_{i}^{A} \hat{\sigma}_{y}, \\
& \hat{B}_{j}=\cos \varphi_{j}^{B} \hat{\sigma}_{x}+\sin \varphi_{j}^{B} \hat{\sigma}_{y}, \\
& \hat{C}_{k}=\cos \varphi_{k}^{C} \hat{\sigma}_{x}+\sin \varphi_{k}^{C} \hat{\sigma}_{y},
\end{aligned}
$$

and so on for all parties; $\hat{\sigma}_{x, y}$ denote the Pauli matrices.

With this choice of measurements and the state (2), it follows that (see, e.g., [25] for details)

$$
P\left(A_{i} B_{j} C_{k} \cdots\right)=\frac{1+v \cos \left(\varphi_{i}^{A}+\varphi_{j}^{B}+\varphi_{k}^{C}+\cdots\right)}{2^{n}} .
$$

Next, let us further simplify the structure of the measurement by choosing the $m$ angles to be evenly distributed around the equator of the Bloch sphere, such that $\varphi_{i}^{A}=\varphi_{i}^{B}=\varphi_{i}^{C}=$ $2 \pi i / m+\pi / n$. With this choice we get

$$
P\left(A_{i} B_{j} C_{k} \cdots\right)=\frac{1-v \cos \left[\frac{2 \pi}{m}(i+j+k+\cdots)\right]}{2^{n}} .
$$

Finally, since the GHZ state has no $(n-1)$ subcorrelations for equatorial measurements, it follows that all $(n-1)$-particle joint probabilities involved in our inequality take the value $1 / 2^{n-1}$, independently of $v$.

\section{THRESHOLD EFFICIENCIES FOR REASONABLE NUMBER OF PARTIES AND MEASUREMENTS}

All observers detect their particles with the same limited efficiency $\eta$. In case of nondetection, they agree to output -1 . Hence the measurement outputs are still binary and the Bell inequality (1) can be used. However the probabilities must be modified in the following way: $P\left(A_{i} B_{j} C_{k} \cdots\right) \rightarrow \eta^{n} P\left(A_{i} B_{j} C_{k} \cdots\right)$ for $n$-party joint probabilities and $P\left(B_{j} C_{k} \cdots\right) \rightarrow \eta^{n-1} P\left(B_{j} C_{k} \cdots\right)$ for $(n-1)$ party joint probabilities.

Bell inequality (1) is now violated whenever

$$
\left[y-\frac{(1-v) x}{m}\right]\left(\frac{\eta m}{2}\right)^{n}-n\left(\frac{\eta m}{2}\right)^{n-1}>0,
$$

where we have used the fact that $\sum_{i, j, k, \ldots=0}^{m-1} \cos \left[\frac{2 \pi}{m}(i+j+\right.$ $k+\cdots)]=0$. From Eq. (6) the threshold efficiency is then found to be

$$
\eta>\eta^{*}=\frac{2 n}{m y-(1-v) x} .
$$

Thus, in order to determine $\eta^{*}$ for any given number of parties $n$ and measurements $m$, we must determine the parameters $x$ and $y$ of the Bell inequality (1) such that the local bound is 0 . We shall see that, in general, the values of $x$ and $y$ leading to the lowest value of $\eta^{*}$ may depend on the visibility $v$ of the state.

We recall first that in order to find the maximal value of a linear Bell polynomial [such as (1)] it is sufficient to consider local deterministic strategies. For commodity we denote by $a_{i}, b_{j}, c_{k}$, and so on the probabilities of getting outcome +1 for measurement $A_{i}, B_{j}, C_{k}$, and so on. We now impose the following condition:

$$
\begin{aligned}
& \sum_{i, j, k, \ldots=0}^{m-1} a_{i} b_{j} c_{k} \cdots\left(y-x \delta_{(i+j+k+\cdots) \bmod m}^{0}\right)-\sum_{j, k, \ldots=0}^{m-1} b_{j} c_{k} \cdots \\
& \quad-\sum_{i, k, \ldots=0}^{m-1} a_{i} c_{k} \cdots-\sum_{i, j, \ldots=0}^{m-1} a_{i} b_{j} \cdots-\cdots \leqslant 0
\end{aligned}
$$

for any deterministic model that is for any $a_{i}, b_{j}, c_{k}, \ldots \in$ $\{0,1\}$. Note first that whenever one (or more) parties outputs -1 for all his measurements (say $a_{i}=0$ for all $i$ ), then the above condition is indeed satisfied since only the second sum may be nonzero. Hence, we can assume that $\alpha \equiv \sum_{i=0}^{m-1} a_{i}>0$, $\beta \equiv \sum_{j=0}^{m-1} b_{j}>0, \gamma \equiv \sum_{i=0}^{m-1} c_{k}>0$, and so on. Condition (8) can then be rewritten as

$$
y \leqslant p+q x,
$$

where $p=\alpha^{-1}+\beta^{-1}+\gamma^{-1}+\cdots$ and $q=S /(\alpha \beta \gamma \cdots)$ and

$$
S \equiv \sum_{i, j, k, \ldots=0}^{m-1} a_{i} b_{j} c_{k} \cdots \delta_{(i+j+k+\cdots) \bmod m}^{0}
$$

For each choice of $a_{i}, b_{j}, c_{k}, \ldots$, condition (9) is a linear constraint between $x$ and $y$, and hence defines a straight line (with positive or zero slope) in a plane with coordinates $x$ and $y$. For finite values of $m$ and $n$ we get a finite set of these lines. To ensure that the local bound of Bell inequality (1) is not greater than $0, x$ and $y$ must be chosen such that the point 

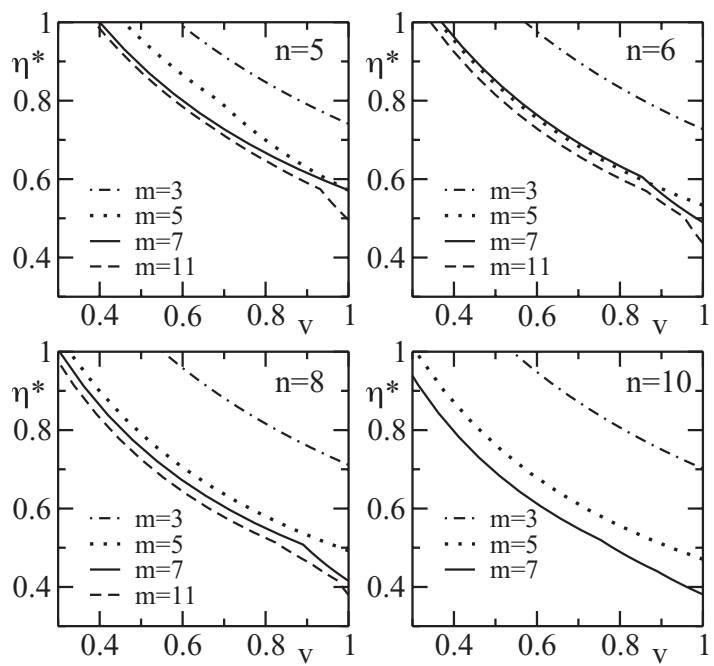

FIG. 1. Threshold efficiency $\eta^{*}$ required given an $n$-qubit GHZ state with visibility $v$. Each observer has $m$ measurement settings.

$(x, y)$ lies below all possible straight lines. For small values of $m$ and $n$ we could find the complete set of straight lines by exhaustive search. We observed that, although in general the number of different lines may be very large, only few of them are relevant for the present problem (see the Appendix for examples and Ref. [26]).

We are now ready to present our main results, namely, the threshold detection efficiencies $\eta^{*}$ for our Bell tests considering all number of parties and measurements which maybe reasonable from an experimental viewpoint. Up to $n=10$ parties and $m=11$ measurements we could determine, for any value of the visibility $v$, the optimal Bell inequality (given by $x$ and $y$ ) leading to the lowest efficiency $\eta^{*}$ (see Fig. 1 and [26]). Notably, efficiencies below $38 \%$ can be reached for a $n=8$ and $m=11$. Also, $\eta^{*}<50 \%$ can be obtained for a 5-qubit GHZ state with $m=11$ (for $n=8, m=5$ is sufficient). Perhaps even more importantly, these threshold efficiencies appear to be robust to noise. In most cases, adding $10 \%$ noise increases $\eta^{*}$ by only a few percent. For instance, for $n=8$ and $m=11$, one has $\eta^{*}<50 \%$ even for $v$ as low as $85 \%$. This shows that multipartite Bell test can tolerate significantly lower detection efficiencies, even in the presence of noise, compared to all bipartite Bell test proposed so far with a reasonable number of measurements and dimensions. Finally, note that we focused here on cases in which $m$ takes prime values; other cases are much less favorable.

\section{ASYMPTOTIC LIMIT}

From a theoretical point of view it is also interesting to investigate the behavior of $\eta^{*}$ in the asymptotic limit, that is, for $n$ and/or $m$ large.

The main difficulty consists in deriving the optimal parameters $x$ and $y$ for Bell inequalities (1) for arbitrary $n$ and $m$. Although we have not been able to find a general solution, we could solve this problem by considering only a subset of all deterministic strategies which we conjecture to be optimal. From our investigation for small values of $m$ (prime) and $n$, we observed that the set of relevant straight lines, delimiting the region of allowed values of $x$ and $y$, are always given by deterministic strategies with a simple and regular structure. Such strategies, which from now on we term "regular arrangements," are as follows. For all parties but one (say A), the output will be +1 for the measurements of lowest indices and -1 for the remaining ones; more formally, $b_{j}=1$ iff $j<\beta, c_{k}=1$ iff $k<\gamma$, and so on. For party A we consider strategies of the form $a_{i}=1$ iff $i=i_{0}, \ldots,\left(\alpha+i_{0}\right) \bmod m$ for $i_{0}=0, \ldots, m-1$. Moreover, it turns out that it is enough to consider strategies in which $\alpha, \beta, \gamma, \ldots$ differ from each other by at most one.

We start by considering the case of a pure $n$-qubit GHZ state, that is, $v=1$. In this case, the threshold efficiency depends only on the parameter $y$ [see Eq. (7)]. As we would like to choose $y$ as large as possible, we are looking for the straight line of the form (9) with zero slope, that is, with $S=0$. For regular arrangements we can derive the optimal efficiencies $\eta^{*}$ for arbitrary $m$ (prime) and $n$. Note first that, for a regular arrangement to achieve $S=0$, the total number of measurements for which the outcome is +1 must be upper bounded: $\alpha+\beta+\gamma+\cdots \leqslant n+m-2$. To see this, consider first the case $\beta=\gamma=\cdots=1$, that is, $b_{j}=c_{k}=\cdots=1$ iff $j=k=\cdots=0$. To ensure that $S=0$, one must choose $a_{0}=0$, which leads to $\alpha \leqslant m-1$, hence finally to $\alpha+\beta+\gamma+\cdots \leqslant n+m-2$. If we then increase $\beta$ by one, we must now also impose that $a_{m-1}=0$, hence decreasing $\alpha$ by one. Thus the total number of measurements with outcome +1 does not increase. Next, one has to maximize the value of $p$ [see Eq. (9)]. Given that the total number of measurements with outcome +1 is upper bounded, we get the largest value of $p$ by distributing these measurements as evenly as possible between the $n$ observers. Hence we get $\alpha=\beta=\gamma=\cdots=(m+n-2) / n$, leading to $y \leqslant y_{\max }=$ $n^{2} /(m+n-2)$; for simplicity we have assumed here that $m-2$ is a multiple of $n$. This leads to the threshold efficiency

$$
\eta^{*}=\frac{2}{n}+\frac{2}{m}-\frac{4}{m n} .
$$

Hence Bell inequality (1) can be violated using detectors with arbitrarily low efficiency $\eta>0$, by choosing $n$ and $m$ large enough. Note that if either $n$ or $m$ is finite, $\eta^{*}$ tends to a strictly positive value. Note also that for any given number of parties $n$, we have that $\eta \rightarrow 2 / n$, for sufficiently large $m$. This improves on the results of Ref. [14], which had $\eta \rightarrow 8 / n$. Finally, note that we have again considered only $m$ prime. For $m$ not prime it is possible to have $\alpha+\beta+\gamma+\cdots>n+m-2$, such that $S=0$.

Next, we investigate the case in which the visibility of the state is limited, that is, $0<v<1$, and give evidence that our Bell tests can tolerate arbitrarily low detection efficiencies even in the presence of an arbitrarily large amount of noise, when taking $n$ and $m$ large enough. We first determined for $n=m \leqslant 59$ the optimal Bell inequalities (i.e., parameters $x, y)$, assuming that the optimal local deterministic strategy is a regular arrangement. The results, shown in Fig. 2, support qualitatively our above claim.

Then we consider the case $m \ll n$. As we could not derive the complete set of conditions on $x$ and $y$, we focused our efforts, as in the noiseless case, on the horizontal line, that is, $y=y_{\max }=n+1-m / 2$ (for $m \ll n$ ). From Eq. (7) one can 


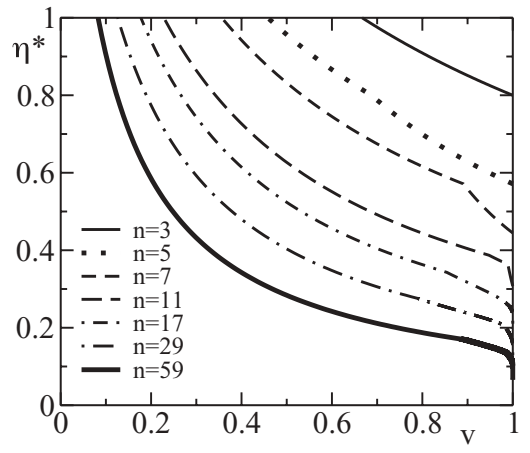

FIG. 2. Threshold detector efficiency $\eta^{*}$ versus the visibility $v$ of the GHZ state, in the case $m=n$. This indicates that in the limit of large $n$ and $m, \eta^{*}$ can become arbitrarily small, even for small visibilities $v$.

see that $x$ must be taken as small as possible in order to get the lowest values of $\eta^{*}$. Hence our goal here is to determine the smallest possible value of $x$, that is, $x_{\min }$, such that all conditions (9) hold. We conjecture that $x_{\min } \leqslant n m$ holds for $m \ll n$, leading to a threshold efficiency

$$
\eta^{*} \simeq \frac{2}{m v} .
$$

Hence, even in the case of arbitrarily small visibility $v, \eta^{*}$ can become arbitrarily small by taking $m \ll n$ large enough. To support our conjecture that $x_{\min } \leqslant n m$, we checked that, for $m \leqslant 199$ (prime) and $n \leqslant 199, x_{\min }$ is always achieved by only two possible strategies: (i) All parties output +1 for all measurements, that is, $\alpha=\beta=\gamma=\cdots=m$, leading to $p=n / m, S=m^{n-1}$, and $q=1 / m$; this corresponds to a line reaching $y=y_{\max }$ at $x_{1}=m y_{\max }-n$; or (ii) a regular arrangement with $\alpha+\beta+\gamma+\cdots=m+n$, leading to $p=$ $n-m / 2, S=2$, and $q=1 / 2^{m-1}$; this corresponds to a line reaching $y=y_{\max }$ at $x_{2}=\left(y_{\max }-p\right) / q=2^{m-1}$, independent of $n$. Indeed $x_{1}, x_{2} \leqslant n m$ when $m \ll n$.

\section{CONCLUSION}

We presented a family of multipartite Bell tests and derived the minimal detection efficiencies required in order to close the detection loophole. Notably, efficiencies below $50 \%$ can be tolerated for a reasonable number of parties and measurements, even in the presence of significant amount of noise. Our Bell tests are based on $n$-qubit GHZ states, which have been realized experimentally. In particular, Ref. [22] recently reported 8qubit GHZ entanglement, with fidelities of $\sim 70 \%$. This would require a detection efficiency of $\sim 60 \%$ in our Bell tests, which seems within reach of current photonic experiments [5]. However, the main challenge is to achieve a heralded preparation of the GHZ state [27]. Nevertheless, this shows that the multipartite setting offers possibilities for a loophole-free Bell test based on photons. More generally, we believe that
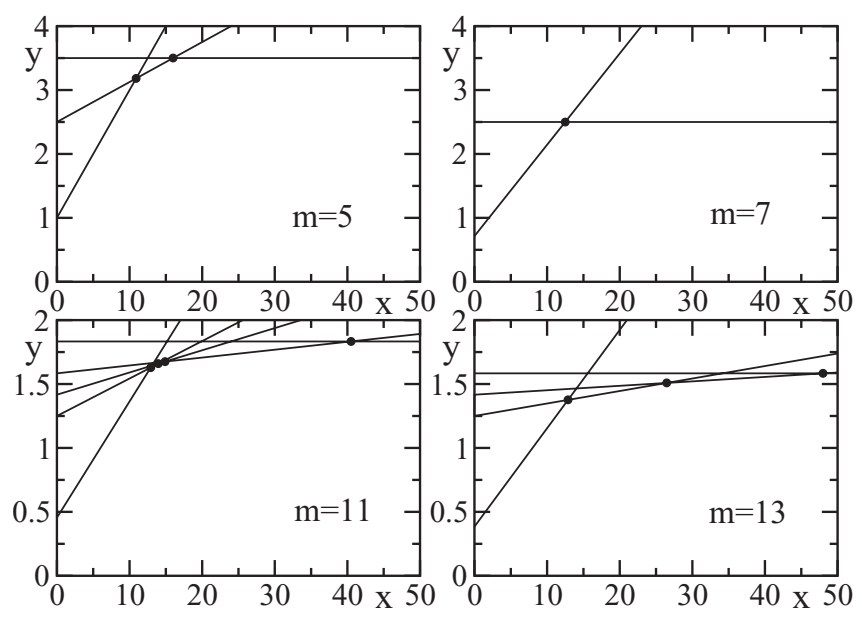

FIG. 3. The set of straight lines defining the region of allowed parameters $x$ and $y$. Each straight line corresponds to one condition as given in Eq. (9) (with equality). Hence all points $(x, y)$ located below all lines will satisfy all conditions (9). Hence for any such choice of parameters $x$ and $y$, the local bound of the Bell inequality will be smaller or equal to 0 as desired. Here we have $n=5$ parties. Only the relevant lines are shown.

our findings open interesting experimental perspectives for multipartite nonlocality, and for its applications $[8,28]$.

\section{ACKNOWLEDGMENTS}

The authors thank C. Simon for discussions, and acknowledge financial support from the UK EPSRC, the Swiss National Science Foundation (Grant PP00P2_138917), the EU DIQIP, the Hungarian National Research Fund OTKA (PD101461), a János Bolyai Grant of the Hungarian Academy of Sciences, and the TÁMOP-4.2.2.C-11/1/KONV-2012-0001 project. The project has also been supported by the European Union, co-financed by the European Social Fund.

\section{APPENDIX}

Here we give more details concerning the choice of parameters $x$ and $y$, defining our Bell inequalities (1), such that the local bound is 0 . As explained in the main text, one must check a finite set of conditions, of the form (9), which define straight lines in the plane with coordinates $x$ and $y$. For small values of $n$ and $m$ the complete set of lines can be found. Figure 3 illustrates the situation for the case of $n=5$ observers, and up to $m=13$ measurements. Note that although the total number of lines is large, only a few turn out to be relevant. Also the optimal choice of $x$ and $y$, which may depend on the visibility of the state $v$ [see Eq. (7)], is always one of the intersections of two (or more) lines (marked by dots in Fig. 3). The $(x, y)$ pairs, along with the ranges of visibilities where they are optimal choice, are shown in Ref. [26]. These values have been used to generate the pieces of the curves shown in Fig. 1.
[1] J. S. Bell, Physics 1, 195 (1964).

[2] A. Aspect, Nature (London) 398, 189 (1999).
[3] D. Cavalcanti, N. Brunner, P. Skrzypczyk, A. Salles, and V. Scarani, Phys. Rev. A 84, 022105 (2011); A. Cabello and F. Sciarrino, Phys. Rev. X 2, 021010 (2012); J. B. Brask 
and R. Chaves, Phys. Rev. A 86, 010103 (2012); C. Teo et al., arXiv:1206.0074; Y. Lim, M. Paternostro, J. Lee, M. Kang, and H. Jeong, Phys. Rev. A 85, 062112 (2012).

[4] J. Hofmann et al., Science 337, 72 (2012).

[5] B. Wittmann et al., New J. Phys. 14, 053030 (2012); D.-H. Smith et al., Nat. Commun. 3, 625 (2012); A. J. Bennett et al., Phys. Rev. X 2, 031003 (2012).

[6] P. M. Pearle, Phys. Rev. D 2, 1418 (1970); see also C. Branciard, Phys. Rev. A 83, 032123 (2011).

[7] M. A. Rowe et al., Nature (London) 409, 791 (2001).

[8] H. Buhrman, R. Cleve, S. Massar, and R. de Wolf, Rev. Mod. Phys. 82, 665 (2010).

[9] A. Acin, N. Brunner, N. Gisin, S. Massar, S. Pironio, and V. Scarani, Phys. Rev. Lett. 98, 230501 (2007).

[10] S. Pironio et al., Nature (London) 464, 1021 (2010); R. Colbeck, Ph.D. thesis, University of Cambridge, 2006; R. Colbeck and A. Kent, J. Phys. A: Math. Th. 44, 095305 (2011).

[11] I. Gerhardt, Q. Liu, A. Lamas-Linares, J. Skaar, V. Scarani, V. Makarov, and C. Kurtsiefer, Phys. Rev. Lett. 107, 170404 (2011); E. Pomarico et al., New J. Phys. 13, 063031 (2011); D. S. Tasca, S. P. Walborn, F. Toscano, and P. H. Souto Ribeiro, Phys. Rev. A 80, 030101(R) (2009).

[12] P. H. Eberhard, Phys. Rev. A 47, R747 (1993); see also G. Lima, E. B. Inostroza, R. O. Vianna, J. A. Larsson, and C. Saavedra, ibid. 85, 012105 (2012).

[13] T. Vértesi, S. Pironio, and N. Brunner, Phys. Rev. Lett. 104, 060401 (2010).

[14] H. Buhrman, P. Høyer, S. Massar, and H. Röhrig, Phys. Rev. Lett. 91, 047903 (2003).

[15] A. Cabello, D. Rodriguez, and I. Villanueva, Phys. Rev. Lett. 101, 120402 (2008).
[16] J.-A. Larsson and J. Semitecolos, Phys. Rev. A 63, 022117 (2001).

[17] R. Chaves and J. B. Brask, Phys. Rev. A 84, 062110 (2011).

[18] Note that in an asymmetric setup, where parties may have different efficiencies (e.g., in atom-photon entanglement), lower efficiencies were reported, see [19].

[19] N. Brunner, N. Gisin, V. Scarani, and C. Simon, Phys. Rev. Lett. 98, 220403 (2007); A. Cabello and J. A. Larsson, ibid. 98, 220402 (2007).

[20] D. M. Greenberger, M. A. Horne, and A. Zeilinger, in Bells Theorem, Quantum Theory, and Conceptions of the Universe, edited by M. Kafatos (Kluwer Academic, Dordrecht, Holland, 1989), pp. 69-72.

[21] C.-Y. Lu et al., Nat. Phys. 3, 91 (2007); R. Prevedel, G. Cronenberg, M. S. Tame, M. Paternostro, P. Walther, M. S. Kim, and A. Zeilinger, Phys. Rev. Lett. 103, 020503 (2009); W. Wieczorek, R. Krischek, N. Kiesel, P. Michelberger, G. Toth, and H. Weinfurter, ibid. 103, 020504 (2009).

[22] X.-C. Yao et al., Nat. Photon. 6, 225 (2012).

[23] W. Dür and J. I. Cirac, Phys. Rev. A 61, 042314 (2000).

[24] R. F. Werner and M. M. Wolf, Phys. Rev. A 64, 032112 (2001).

[25] K. F. Pál and T. Vértesi, Phys. Rev. A 83, 062123 (2011).

[26] For all data, see http://www.atomki.hu/atomki/TheorPhys/ Deteff_GHZ/BellXY.html.

[27] P. Walther, M. Aspelmeyer, and A. Zeilinger, Phys. Rev. A 75, 012313 (2007).

[28] V. Scarani and N. Gisin, Phys. Rev. Lett. 87, 117901 (2001); J. Silman, A. Chailloux, N. Aharon, I. Kerenidis, S. Pironio, and S. Massar, ibid. 106, 220501 (2011); L. Aolita, R. Gallego, A. Cabello, and A. Acin, ibid. 108, 100401 (2012). 